\title{
A Finger-Like Projection in the Carotid Artery: A Rare Source of Embolic Stroke Requiring Carotid Endarterectomy
}

\author{
Neal S. Gerstein, MD FASE ${ }^{1}$ \\ Liem C. Nguyen, $M D^{2}$ \\ Omar S. Akbik, $M D^{3}$ \\ Howard Yonas, $M D^{3}$ \\ Andrew P. Carlson, MD MS-CR ${ }^{3}$ \\ ${ }^{1}$ Department of Anesthesiology and Critical Care Medicine and ${ }^{3}$ Department of \\ Neurosurgery \\ School of Medicine \\ University of New Mexico \\ Albuquerque, NM USA \\ ${ }^{2}$ Department of Anesthesiology \\ University of California - San Diego Medical Center and Sulpizio Cardiovascular Center \\ San Diego, CA USA
}

\begin{abstract}
Atherosclerotic lesions of the extracranial carotid arteries are one of the most common cases of stroke. Rarely, a stroke may result from isolated non-stenotic carotid disease in the absence of systemic manifestations of cardiovascular disease or significant cardiovascular risk factors. We present an unusual case of multiple strokes resulting from a solitary finger-like projection within the posterior wall of the carotid artery in an otherwise healthy patient. This small finger-like projection has a propensity to act as a nidus for thrombus formation and a potential source of cerebral embolism.

\section{Introduction}

Atherosclerotic lesions of the extracranial carotid arteries are one of the most common causes of stroke. Intervention, whether it be via an open or endovascular technique, is typically reserved for symptomatic patients with moderate to severe carotid stenosis while intervention in asymptomatic patients is less clear $(1,2)$. However, in rare cases, a cerebrovascular accident (CVA) may result from isolated non-stenotic carotid disease in healthy patients in the absence of systemic manifestations of cardiovascular disease or significant cardiovascular risk factors. CVA as a result from isolated carotid artery disease has not been previously described in the anesthesiology literature. We present an unusual case of CVA resulting from a solitary finger-like projection within the wall of the carotid artery in an otherwise healthy patient. The etiology associated with a CVA in this context relates to a small and minimal posterior carotid plaque that has a propensity to act as a nidus for thrombus formation and a potential source of cerebral embolism.
\end{abstract}


Our case exemplifies this atypical cause of a CVA and heretofore minimally described entity involving the carotid artery system.

\section{Case}

In April 2016, a 44-year-old non-smoking woman with a past medical history solely consisting of well-controlled hypertension and hyperlipidemia was exercising when she developed right-sided weakness. She was diagnosed with an ischemic right-sided CVA in the right middle cerebral artery territory. Her symptoms spontaneously resolved. She was managed with aspirin and warfarin for six months followed by aspirin monotherapy. In April 2017, she developed nearly identical symptoms, which again resolved with conservative therapy (aspirin and warfarin) and she was referred for neurosurgery consultation and further evaluation.

During her most recent evaluation, aside from a body-mass index of $33 \mathrm{~kg} / \mathrm{m}^{2}$, her physical examination was completely normal including a complete neurologic and cardiac evaluation. Laboratory evaluation revealed no evidence of a hypercoagulable state or sickle-cell disease, autoimmune disease, abnormal erythrocyte sedimentation rate or C-reactive protein levels, and there was no evidence of an intracardiac shunt by transthoracic echocardiography.

Bilateral neck ultrasound duplex scanning revealed normal flow in both the internal carotid and both vertebral arteries. Magnetic resonance angiography of her neck vessels at the time of the initial stroke demonstrated bilateral mild narrowing and a posterior irregularity along with enlargement of the proximal internal carotid artery (ICA) just beyond the bifurcation, which was deemed hemodynamically insignificant (Figure 1).

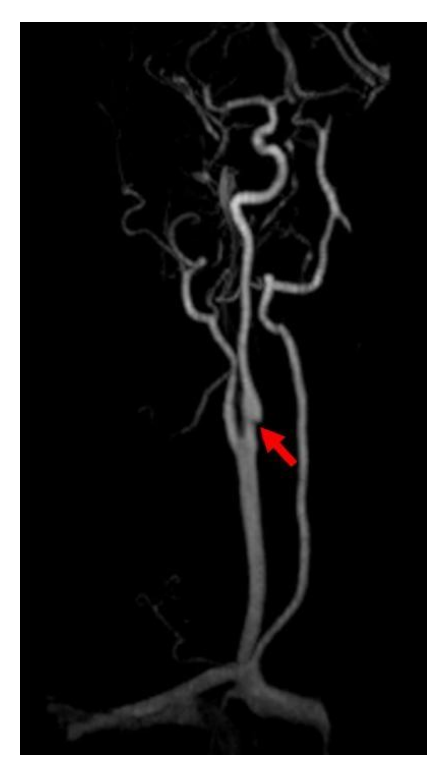

Figure 1. Preoperative magnetic resonance angiography with contrast; Red arrow indicating defect in posterior right internal carotid artery. 
Computed tomographic angiography (CTA) of her neck vasculature performed at the time of the second stroke one year later revealed no significant stenosis of her common carotid, internal carotid, or vertebral arteries but did re-demonstrate a right-sided small finger-like extension in the posterior carotid wall at the level of the bifurcation (Figure 2).

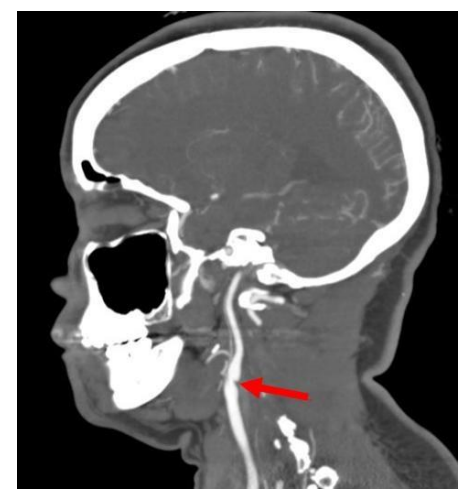

Figure 2. Preoperative computed tomography angiogram. Red arrow indicating right posterior internal carotid artery abnormality.

Anticoagulation was continued for another 4 months and a follow-up CTA did not reveal any change in the previous noted finger-like lesion within the carotid artery.

After evaluation by our neurosurgical colleagues, the decision was made to prepare the patient for a right-sided carotid endarterectomy (CEA). In addition to the routine standard monitors, additional monitoring modalities included invasive arterial blood pressure monitoring, 16-lead electroencephalogram, and bilateral cerebral oximetry monitoring. Her CEA consisted of a longitudinal arteriotomy from the distal common carotid artery into the proximal ICA. The 'finger' of firm, irregular plaque was seen on the posterior ICA wall and could be easily dissected off the wall, ruling out a congenital web. The plaque was neither soft nor ruptured at the time of surgery; it was an irregular finger-like extension from the underlying plaque that was presumably the focus of thrombus formation (Figure 3).

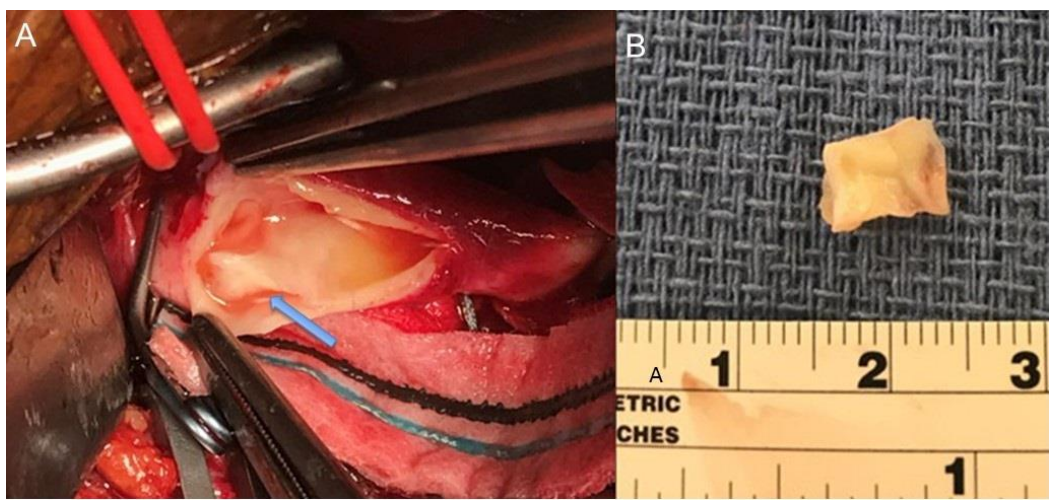

Figure 3. Panel A: blue arrow indicating finger-like abnormal projection from posterior wall of right internal carotid artery. Panel B: excised projection specimen. 
Pathological examination of the plaque revealed no evidence of gross calcifications, no signs of microscopic ulceration, or intra-plaque hemorrhage that are associated with an unstable plaque. The ICA clamp was then released temporarily to allow backflow of blood and with it washout of plaque that might have migrated upstream. Once the arteriotomy closure was complete, vascular clamps were removed and satisfactory pulsations were noted in the common carotid artery and external carotid artery, as well as the ICA. Following an uneventful emergence from anesthesia, the patient was extubated and brought to ICU in stable condition. Postoperative CTA demonstrated a normal caliber and lumen in the surgically treated right carotid artery (Figure 4).

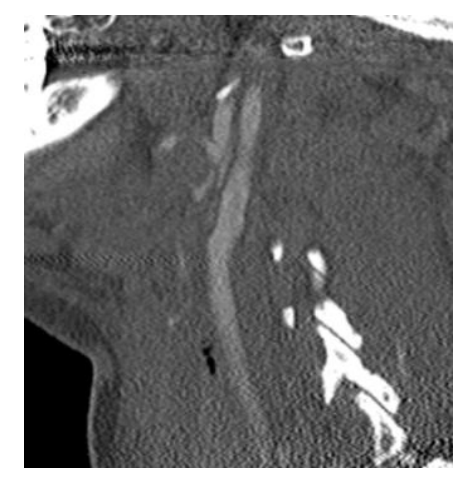

Figure 4. Postoperative computed tomography angiogram demonstrating normal right internal carotid artery lumen.

\section{Discussion}

Stroke is a leading cause of death in developed nations with a majority of those ischemic in nature. Extracranial carotid artery atherosclerotic disease is the third leading cause of ischemic stroke in the general population (3). While medical management including antiplatelet therapy, treatment of hypertension, hyperlipidemia, diabetes, and smoking cessation have been shown to decrease the risk of stroke, surgical intervention in the form of CEA has been widely investigated in several randomized control studies and has proven efficacy in the appropriate patient population. A 2017 Cochrane systematic review along with other robust reviews found CEA most effective in symptomatic patients with $>70 \%$ and is of some benefit for patients with $50-69 \%$ symptomatic stenosis $(4,5)$. Surgery plays a limited role in complete or near complete occlusion (6).

While the above trials deal primarily with symptomatic carotid stenosis, a different pathology known as free-floating thrombus (FFT) can exist with or without carotid stenosis. As in our case, these patients are typically younger patients without established peripheral vascular disease or other systemic cardiovascular diseases. They typically have underlying atherosclerotic disease that predisposes them to thromboembolic events and as such are at a high risk for recurrent ischemic strokes. Most studies show that patients with FFT who are treated medically with anticoagulation have complete dissolution of the FFT without any further neurologic progression $(7,8)$. 
In contrast to FFT, our case patient was found to have a finger-like projection from the posterior wall of the right ICA just distal to its bifurcation without evidence of luminal thrombus. Our patient had persistent ischemic CVAs despite therapeutic anticoagulation and antiplatelet therapy with warfarin and aspirin, respectively. She continued to have persistent imaging findings of a small finger like projection on repeat neck CTAs. Intraoperatively, no thrombus was identified but rather a small plaque was resected which appeared to be similarly shaped to the finger-like projection seen on her CTA with an irregular intraluminal surface thought to be the nidus for thrombus formation. Upon further examination of the plaque by pathology, no gross calcifications were identified. Histologically no signs of microscopic ulceration or intraplaque hemorrhage were identified to indicate an unstable plaque, which is more commonly seen in advanced atherosclerotic disease. Critical differences in plaque morphology have been found to highly correlate with whether a patient has symptomatic or asymptomatic carotid disease (9).

There is a single similar case from 2011 from our institution, describing a 48-year-old woman who presented with intermittent hand numbness, facial weakness, and dysarthria (10). CTA of her head and neck demonstrated a several millimeter protrusion from her posterior ICA just distal to the bifurcation. The patient had recurrent neurologic symptoms attributed to ongoing cerebral emboli despite anticoagulation and antiplatelet requiring CEA, during which organized thrombus was found in continuity with her isolated thin $(1 \mathrm{~mm})$ posterior carotid artery atherosclerotic plaque. It was concluded that the development of significant neurologic symptoms in the context of minimal stenosis is due to carotid endothelial hyperplasia with organizing thrombus on top of a small preexisting carotid atherosclerotic plaque. Similarly, our case report illustrates a patient receiving maximal medical therapy in the form of warfarin anticoagulation and antiplatelet therapy with persistent ischemic CVAs and an intraluminal plaque. The previous case had evidence of organizing thrombus while our case demonstrated only irregular plaque. This could either be because any adherent thrombus was washed out during the opening or that the thrombus itself had resolved with prolonged anticoagulation, leaving the finger-like plaque in the lumen. This speaks to a different pathology than what is typically observed in patients with FFT in that this intraluminal plaque morphology itself likely places the patient at risk for recurrent thrombus formation.

In summary, our rare etiology of stroke is heretofore unreported in the perioperative medicine literature. This case illustrates that in an otherwise healthy patient without systemic cardiovascular disease, the possibility of significant but minimal isolated carotid disease may be a nidus for thrombus and ultimately an embolic etiology for a significant neurologic injury. This report, along with the similar case described by Tran and Yonas (10), do not indicate a clear causal relationship. However, it is plausible that the described recurrent ipsilateral strokes are related to these uncommon and characteristic carotid morphologic findings. These assertions are further substantiated by the lack of any new symptoms during all patient follow-up visits. Nonetheless, a detailed study to document morphology involving a large sample of similar plaques of otherwise similar size and composition would be needed in order to make a definitive 
conclusion regarding the association between this finger-like carotid projection and recurrent CVAs. Perioperative and critical care physicians need to be aware that advanced radiological imaging is required to identify this isolated carotid pathology. Its association with cerebral emboli should be considered when presented with recurrent CVA events in the context of minimal evidence of atherosclerotic disease on routine carotid screening studies.

\section{References}

1. Kernan WN, Ovbiagele B, Black HR, et al. Guidelines for the prevention of stroke in patients with stroke and transient ischemic attack: a guideline for healthcare professionals from the American Heart Association/American Stroke Association. Stroke. 2014;45(7):2160-236. [CrossRef] [PubMed]

2. Meschia JF, Bushnell C, Boden-Albala B, et al. Guidelines for the primary prevention of stroke: a statement for healthcare professionals from the American Heart Association/American Stroke Association. Stroke. 2014;45(12):3754-832. [CrossRef] [PubMed]

3. Ooi YC, Gonzalez NR. Management of extracranial carotid artery disease. Cardiol Clin. 2015;33(1):1-35. [CrossRef] [PubMed]

4. Orrapin S, Rerkasem K. Carotid endarterectomy for symptomatic carotid stenosis. Cochrane Database Syst Rev. 2017;6:CD001081. [CrossRef] [PubMed]

5. Meschia JF, Klaas JP, Brown RD, et al. Evaluation and Management of Atherosclerotic Carotid Stenosis. Mayo Clin Proc. 2017;92(7):1144-57. [CrossRef] [PubMed]

6. Rothwell PM, Eliasziw M, Gutnikov SA, et al. Endarterectomy for symptomatic carotid stenosis in relation to clinical subgroups and timing of surgery. Lancet. 2004;363(9413):915-24. [CrossRef] [PubMed]

7. Vellimana AK, Kadkhodayan Y, Rich KM, et al. Symptomatic patients with intraluminal carotid artery thrombus: outcome with a strategy of initial anticoagulation. J Neurosurg. 2013;118(1):34-41. [CrossRef] [PubMed]

8. Bhatti AF, Leon LR, Jr., Labropoulos N, et al. Free-floating thrombus of the carotid artery: literature review and case reports. J Vasc Surg 2007;45(1):199-205. [CrossRef] [PubMed]

9. Virmani R, Ladich ER, Burke AP, et al. Histopathology of carotid atherosclerotic disease. Neurosurgery. 2006;59(5 Suppl 3):S219-27; discussion S3-13. [CrossRef] [PubMed]

10. Tran $\mathrm{H}$, Yonas $\mathrm{H}$. Small carotid thrombus and minimal stenosis causing repeated embolic strokes. J Neuroimaging. 2011;21:266-8. [CrossRef] [PubMed] 